\title{
EFFECTS OF LIQUID LOADINGS ON LAMB WAVES IN CONTEXT OF SIZE DEPENDENT COUPLE STRESS THEORY
}

\author{
VIKAS SHARMA \\ Department of Mathematics, Lovely Professional University, Phagwara, Punjab, India \\ e-mail: vikas.sharma@lpu.co.in; vikassharma10a@yahoo.co.in \\ SATish KUMAR \\ School of Mathematics and Computer Applications, Thapar University, Patiala, Punjab, India \\ e-mail: satishk.sharma@thapar.edu
}

\begin{abstract}
For understanding the effects of an internal microstructure, generalised microcontinuum theories with additional microstructural parameters are developed. One such a parameter, called the characteristic length of the material comparable with the internal cell size of the material is involved in the couple stress theory. The problem of propagation of Lamb waves in a plate with an internal microstructure and loaded with an inviscid liquid on both sides is studied using the couple stress theory. The dispersion equation of Lamb waves with the liquid loadings is derived. The impact of the liquid loadings is studied on the propagation of Lamb waves. The effect of the characteristic length is also studied on the phase velocity of Lamb waves in the plate for various modes in the presence of liquid loadings.
\end{abstract}

Keywords: couple stress, characteristic length, Lamb waves, elastic waves, liquid loadings

\section{Introduction}

Classical theory of elasticity is inadequate to capture the size effects, as the atomic structure of the material is ignored in this theory, and it is based on the assumption of homogeneity of the material. It can describe elastic deformations and properties of materials at macroscale. However, experimental evidences show that mechanical behaviour of the material at microscale is different from its behaviour at macroscale. To explain this, there was a need to develop a new theory or to modify existing ones, which can fill this gap of theoretical and experimental results. Microcontinuum theories such as micropolar theory (Eringen, 1968), strain gradient (Mindlin, 1964) and couple stress theory (Toupin, 1962; Mindlin and Tiersten, 1962; Koiter, 1964; Hadjesfandiari and Dargush, 2011) were developed to investigate the microstructural effects in the material.

Voigt (1887) was the first to create the idea of couple stresses in the materials by assuming that an infinitesimal surface element transmits both Cauchy stresses and couple stresses. Cosserat and Cosserat (1909) gave the mathematical model to analyse materials with couple stresses by considering that the deformation of the medium is described by a displacement vector and an independent rotation vector. This theory was not recognised at that time. Later on, this concept was used by many researchers like Toupin (1962), Mindlin and Tiersten (1962), Koiter (1964), Eringen (1968), Nowacki (1974) to explore microstructural effects in the material. Many problems of wave propagation in an elastic medium with a microstructure under different conditions have been studied by applying the couple stress theory. Sengupta and Ghosh (1974) studied the effects of couple stresses on wave propagation in an elastic layer and they observed that couple stresses affect the velocity of propagation of waves in the elastic layer. Das et al. 
(1991) studied thermo-viscoelastic Rayleigh waves under the influence of couple stress and gravity. They derived more general equations of phase velocity for these waves and showed that it reduces to classical elastic Rayleigh waves in the absence of couple stresses, viscosity and gravity. Ottosen et al. (2000) studied Rayleigh waves by applying the indeterminate couple stress theory. Georgiadis and Velgaki (2003) shown the dispersive nature of Rayleigh waves propagating along the surface of a half-space at high frequencies using the couple stress theory and also tried to estimate the values of microstructural parameters in couple stress theory. Akgoz and Civalek (2013) did the modeling and analysis of micro-sized plates resting on an elastic medium using the modified couple stress theory. Chen and Li (2014) proposed a new modified couple stress theory for anisotropic elasticity containing three length scale parameters and developed composite laminated Kirchhoff plate models under this theory.

Hadjesfandiari and Dargush (2011) formulated a couple stress theory for an isotropic material involving three parameters $\lambda, \mu$ and $\eta$. The constants $\lambda, \mu$ have the same meaning as Lamé constants in Cauchy elasticity and $\eta$ is a length scale parameter which accounts for couple stress effects for isotropic solids. Evaluation of $\eta$ requires characteristic material length $l$ which is absent in Cauchy elasticity. One of the major problems in these size dependent elastic theories (Cosseret, 1909), micropolar (Eringen, 1968) and couple stress (Toupin, 1962; Mindlin and Tiersten, 1962; Koiter, 1964; Hadjesfandiari and Dargush, 2011) is determination of these length scale parameters. It was observed (Lakes, 1991) that the characteristic length would be undetectable in any macroscopic mechanical experiment, but have relevance in studies involving composite and cellular solids. In fibrous composites, the characteristic length may be of the order of spacing between the fibres. In cellular solids, it may be comparable to the average cell size of the material.

Lakes et al. (1986), while studying propagation of an ultrasonic wave in wet bone, pointed out that bone may be regarded as a composite with particulate, porous and fibrous structural elements at different levels of scale. Vavva et al. (2009) also described bone as a strongly heterogeneous natural composite with a complex structure and carried out the study by taking the characteristic length comparable to the size of the bone microstructure that is of the order of 10 to $500 \mu \mathrm{m}$.

Lamb waves travel in thin stress free elastic plates and were originally studied by Horace Lamb (1917). These waves are extremely useful for detection of cracks, corrosion and other defects in materials using non destructive testing techniques and have immense applications in aerospace, industrial and engineering fields. Viktorov (1967) gave the further details by providing dispersive nature of Lamb waves and explained that they are formed by interference of multiple reflections and mode conversion of longitudinal and shear waves at the free surfaces of the plate. Osborne and Hart (1945) examined Lamb waves activated in steel plates in underwater explosions and made a comparison between theoretical and experimental results. Schoch (1952) investigated the effect of an inviscid liquid loading on the propagation of Lamb waves and derived the dispersion relation for leaky Lamb waves for an isotropic plate. $\mathrm{Wu}$ and Zhu (1992) studied the propagation of Lamb waves in a plate bordered with inviscid liquid layers on both sides. The dispersion equations of this case were derived and showed that phase velocity varies with thickness of the liquid layers. Sharma and Pathania (2003) studied generalized thermoelastic Lamb waves in a homogeneous isotropic, thermally conducted plate bordered with layers of an inviscid liquid or inviscid half space. Sharma and Kumar (2009) studied Lamb waves in micropolar thermoelastic solid plates immersed in a liquid with varying temperature. They studied the effect of the characteristic length and coupling factor on the phase velocity of the Lamb wave. Vavva et al. (2009) discussed velocity dispersion of guided waves propagating in a free gradient elastic plate by showing its application to cortical bone. They studied microstructural effects on the propagation of guided waves by applying gradient elasticity and concluded that this theory can provide additional information for better understanding of the waves. Wu 
et al. (2009) investigated vibration characteristics in a microscale fluid loaded rectangular isotropic plate attached to a uniformly distributed mass. They studied plate vibrations under the simultaneous effect of fluid loadings and attached mass loadings.

In an earlier work, the authors (2014) successfully employed the couple stress model (Hadjesfandiari and Dargush, 2011) to capture size effects on velocity dispersion in an elastic plate. It was observed that the microstructural parameter affected the dispersion of the Lamb wave. The dynamical characteristics of a structure get affected when it is surrounded by a fluid. This study becomes more important when the material under consideration exhibits internal microstructure. Lamb waves are guided waves propagating in a traction free plate surface, however if the surface of the plate is in contact with the fluid, a part of energy will leak into the liquid, and this phenomenon may find possible applications not only in non destructive evaluation of materials, but also in biomedical field. Keeping this in mind, the fluid-solid model adopted by Sharma and Kumar (2009) is applied to extend the study of Lamb waves (Sharma and Kumar, 2014) in a thin elastic plate with a microstructure. The study is carried out for a thin elastic plate having mechanical properties similar to bone, bordered on both sides with an inviscid liquid by applying the couple stress theory (Hadjesfandiari and Dargush, 2011) to capture the effects of both liquid loading and microstructure in terms of the internal characteristic length of the material.

\section{Formulation and solution of the problem}

Consider an infinite homogeneous isotropic, elastic plate of thickness $2 d$. The plate is bordered both on the top and bottom with infinitely large homogeneous inviscid liquid layers of thickness $H$. Consider the origin of the coordinate system $(x, y, z)$ in the middle of the plate. The $X Y$-plane is chosen to coincide with the middle surface of the plate and $z$-axis is normal to the plate, along the thickness of the plate pointing vertically downwards. We consider the $X Z$-plane as the plane of incidence and assume that the solutions are explicitly independent of $y$.

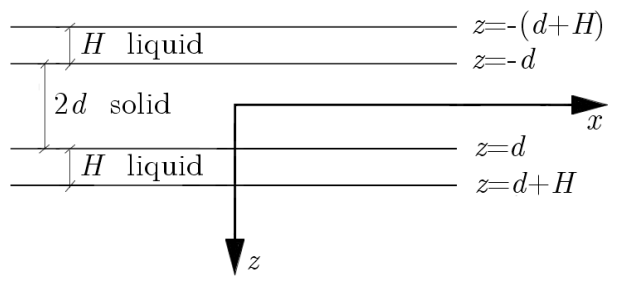

Fig. 1. Geometry of the considered structure

The basic governing equation of motion and constitutive relations of couple stress elasticity for an isotropic material in the absence of body forces (Hadjesfandiari and Dargush, 2011) are given by

$$
\begin{aligned}
& \left(\lambda+\mu+\eta \nabla^{2}\right) u_{k, k i}+\left(\mu-\eta \nabla^{2}\right) \nabla^{2} u_{i}=\rho \ddot{u}_{i} \\
& \sigma_{j i}=\lambda u_{k, k} \delta_{i j}+\mu\left(u_{i, j}+u_{j, i}\right)-\eta \nabla^{2}\left(u_{i, j}-u_{j, i}\right) \\
& \mu_{j i}=4 \eta\left(\omega_{i, j}-\omega_{j, i}\right) \quad \omega_{i}=\frac{1}{2} \epsilon_{i j k} u_{k, j}
\end{aligned}
$$

where $\lambda$ and $\mu$ are Lamé constants, $\eta=\mu l^{2}$ is the couple-stress coefficient, $l$ is the characteristic length, $\rho$ is the density of the plate, $u_{i}$ are the displacement components, commas are used for partial differentiation and dot notation $(\cdot)$ is used for partial differentiation with time. Here, $\sigma_{j i}$ is the non-symmetric force-stress tensor, $\mu_{j i}$ is skew symmetric couple-stress tensor, $\omega_{i}$ is the rotation vector, $\delta_{i j}$ is Kronecker's delta and $\epsilon_{i j k}$ is the permutation tensor. 
In the solid, we take

$$
u=\frac{\partial \phi}{\partial x}-\frac{\partial \psi}{\partial z} \quad w=\frac{\partial \phi}{\partial z}+\frac{\partial \psi}{\partial x}
$$

where $u$ and $w$ are the $x$ and $z$ components of the particle displacement in the plate. $\phi$ and $\vec{\psi}=(0, \psi, 0)$ are the potential functions of the longitudinal and shear waves in the solid. In the liquid boundary layers, we have

$$
\begin{array}{ll}
u_{1}^{\prime}=\frac{\partial \phi_{1}}{\partial x}-\frac{\partial \psi_{1}}{\partial z} & w_{1}^{\prime}=\frac{\partial \phi_{1}}{\partial z}+\frac{\partial \psi_{1}}{\partial x} \\
u_{2}^{\prime}=\frac{\partial \phi_{2}}{\partial x}-\frac{\partial \psi_{2}}{\partial z} & w_{2}^{\prime}=\frac{\partial \phi_{2}}{\partial z}+\frac{\partial \psi_{2}}{\partial x}
\end{array}
$$

where $\phi_{j}$ and $\psi_{j}, j=1,2$ are the scalar potential and vector potential for the bottom liquid layer $(j=1)$ and for the top liquid layer $(j=2), u_{j}^{\prime}$ and $w_{j}^{\prime}$ are respectively $x$ and $z$ components of the particle displacement in the layers of the liquid. Because the inviscid liquid does not support the shear motion, so the shear modulus of the liquid vanishes and hence $\psi_{j}=0, j=1,2$. The potential functions $\phi, \psi$ and $\phi_{j}$ satisfy the basic governing equations

$$
\nabla^{2} \phi=\frac{1}{C_{1}^{2}} \frac{\partial^{2} \phi}{\partial t^{2}} \quad \nabla^{2} \psi-l^{2} \nabla^{4} \psi=\frac{1}{C_{2}^{2}} \frac{\partial^{2} \psi}{\partial t^{2}} \quad \nabla^{2} \phi_{j}=\frac{1}{C_{L}^{2}} \frac{\partial^{2} \phi_{j}}{\partial t^{2}}
$$

and for the liquid the stresses are $\sigma_{j i}^{\prime}=\lambda_{L} u_{k, k}^{\prime} \delta_{i j}$ and the couple stresses in the case of the liquid are all zero. $C_{1}^{2}=(\lambda+2 \mu) / \rho, C_{2}^{2}=\mu / \rho$ are the dilatational and shear wave speeds, respectively, in the classical theory of elasticity $C_{L}^{2}=\lambda_{L} / \rho_{L}$. Here $C_{L}$ is the velocity of sound in the liquid and $\lambda_{L}$ is the bulk modulus. Prime denotes the same quantities for the liquid layer.

Using equations (2.2) and (2.3) in equations (2.1) $)_{2,3}$, we get stresses and couple stresses in terms of the potential functions as

$$
\begin{aligned}
\sigma_{z x} & =\mu\left(\frac{\partial^{2} \psi}{\partial x^{2}}-\frac{\partial^{2} \psi}{\partial z^{2}}+2 \frac{\partial^{2} \phi}{\partial x \partial z}\right)+\mu l^{2}\left(\frac{\partial^{4} \psi}{\partial x^{4}}+\frac{\partial^{4} \psi}{\partial z^{4}}+2 \frac{\partial^{4} \psi}{\partial x^{2} \partial z^{2}}\right) \\
\sigma_{z z} & =\mu\left[\frac{C_{1}^{2}}{C_{2}^{2}}\left(\frac{\partial^{2} \phi}{\partial x^{2}}+\frac{\partial^{2} \phi}{\partial z^{2}}\right)-2 \frac{\partial^{2} \phi}{\partial x^{2}}+2 \frac{\partial^{2} \psi}{\partial x \partial z}\right] \\
\mu_{z y} & =-2 \mu l^{2}\left(\frac{\partial^{3} \psi}{\partial z^{3}}+\frac{\partial^{3} \psi}{\partial z \partial x^{2}}\right)
\end{aligned}
$$

and the value of stresses in the liquid in terms of the potential function becomes

$$
\sigma_{z z}^{\prime}=\lambda_{L}\left(\frac{\partial^{2} \phi_{j}}{\partial x^{2}}+\frac{\partial^{2} \phi_{j}}{\partial z^{2}}\right)
$$

We assume solutions of the form

$$
\left\{\phi, \psi, \phi_{j}\right\}=\left\{f(z), g(z), \bar{\phi}_{j}(z)\right\} \mathrm{e}^{\mathrm{i} \xi(x-c t)}
$$

where $c=\omega / \xi$ is the phase velocity, $\omega$ is the frequency and $\xi$ is the wave number. Using solutions (2.7) in Eqs. (2.4) and solving the resulting differential equations, we get the expressions for $\phi$, $\psi$ and $\phi_{j}$ as

$$
\begin{array}{ll}
\phi=\left(A_{1} \cos \alpha z+A_{2} \sin \alpha z\right) \mathrm{e}^{\mathrm{i} \xi(x-c t)} & \\
\psi=\left(A_{3} \cos \beta z+A_{4} \sin \beta z+A_{5} \cos \gamma z+A_{6} \sin \gamma z\right) \mathrm{e}^{\mathrm{i} \xi(x-c t)} \\
\phi_{1}=A_{7} \sin \delta[z-(d+H)] \mathrm{e}^{\mathrm{i} \xi(x-c t)} & d<z<d+H \\
\phi_{2}=A_{8} \sin \delta(z+d+H) \mathrm{e}^{\mathrm{i} \xi(x-c t)} & -(d+H)<z<-d
\end{array}
$$


where

$$
\begin{array}{ll}
\alpha^{2}=\xi^{2}\left(\frac{c^{2}}{C_{1}^{2}}-1\right) & \beta^{2}+\gamma^{2}=-\left(2 \xi^{2}+\frac{1}{l^{2}}\right) \\
\beta^{2} \gamma^{2}=\frac{\xi^{2}}{l^{2}}\left(1+\xi^{2} l^{2}-\frac{c^{2}}{C_{2}^{2}}\right) & \delta^{2}=\xi^{2}\left(\frac{c^{2}}{C_{L}^{2}}-1\right)
\end{array}
$$

Here $\phi_{1}$ and $\phi_{2}$ are solutions of standing waves and are chosen in such a way that the acoustical pressure is zero at $z= \pm(d+H)$.

The boundary conditions at the solid-liquid interfaces $z= \pm d$ to be satisfied are:

(i) The magnitude of the normal component of the stress tensor of the plate should be equal to the pressure of the liquid, that is $\sigma_{z z}=\sigma_{z z}^{\prime}$

(ii) Since the liquid does not support the shear motion so, the tangential component of the stress tensor should be equal to zero, which implies $\sigma_{z x}=0$

(iii) Couple stress tensor, $\mu_{z y}$ should vanish, that is $\mu_{z y}=0$

(iv) Normal component of the displacement of the solid should be equal to that of the liquid, that is $w=w_{j}^{\prime}$ where $j=1,2$

Imposing these above mentioned boundary conditions and using Eqs. (2.8), we get the following four equations

$$
\begin{aligned}
& \mu\left[\left(-\frac{C_{1}^{2}}{C_{2}^{2}}\left(\xi^{2}+\alpha^{2}\right)+2 \xi^{2}\right) C_{11} A_{1}+\left(-\frac{C_{1}^{2}}{C_{2}^{2}}\left(\xi^{2}+\alpha^{2}\right)+2 \xi^{2}\right) S_{1} A_{2}-(2 \mathrm{i} \xi \beta) S_{2} A_{3}\right. \\
& \left.\quad+(2 \mathrm{i} \xi \beta) C_{22} A_{4}-(2 \mathrm{i} \xi \gamma) S_{3} A_{5}+(2 \mathrm{i} \xi \gamma) C_{3} A_{6}\right]-\lambda_{L}\left(\xi^{2}+\delta^{2}\right) A_{7} S_{4}=0 \\
& \mu\left[(-2 \mathrm{i} \xi \alpha) S_{1} A_{1}+(2 \mathrm{i} \xi \alpha) C_{11} A_{2}+\left(-\xi^{2}+\beta^{2}+l^{2} \xi^{4}+l^{2} \beta^{4}+2 l^{2} \xi^{2} \beta^{2}\right) C_{22} A_{3}\right. \\
& \quad+\left(-\xi^{2}+\beta^{2}+l^{2} \xi^{4}+l^{2} \beta^{4}+2 l^{2} \xi^{2} \beta^{2}\right) S_{2} A_{4}+\left(-\xi^{2}+\gamma^{2}+l^{2} \xi^{4}+l^{2} \gamma^{4}+2 l^{2} \xi^{2} \gamma^{2}\right) C_{3} A_{5} \\
& \left.\quad+\left(-\xi^{2}+\gamma^{2}+l^{2} \xi^{4}+l^{2} \gamma^{4}+2 l^{2} \xi^{2} \gamma^{2}\right) S_{3} A_{6}\right]=0 \\
& \quad-2 \eta\left[\left(\beta^{3}+\xi^{2} \beta\right) S_{2} A_{3}-\left(\beta^{3}+\xi^{2} \beta\right) C_{22} A_{4}+\left(\gamma^{3}+\xi^{2} \gamma\right) S_{3} A_{5}-\left(\gamma^{3}+\xi^{2} \gamma\right) C_{3} A_{6}\right]=0 \\
& -A_{1} \alpha S_{1}+A_{2} \alpha C_{11}+A_{3} \mathrm{i} \xi C_{22}+A_{4} \mathrm{i} \xi S_{2}+A_{5} \mathrm{i} \xi C_{3}+A_{6} \mathrm{i} \xi S_{3}-A_{7} \delta C_{4}=0
\end{aligned}
$$

Here $S_{1}=\sin (\alpha d), S_{2}=\sin (\beta d), S_{3}=\sin (\gamma d), C_{11}=\cos (\alpha d), C_{22}=\cos (\beta d), C_{3}=\cos (\gamma d)$, $S_{4}=\sin (\delta H)$ and $C_{4}=\cos (\delta H)$.

Equations (2.9) will have a non-trivial solution if the determinant of coefficients of the unknowns $A_{1}, A_{2}, A_{3}, A_{4}, A_{5}, A_{6}$ and $A_{7}$ vanishes. After applying this condition to the above system of equations and with a series of tedious mathematical calculations, we obtain the following secular equations for the Lamb waves in a plate loaded with an inviscid liquid layer on both sides

$$
\begin{aligned}
\pm & \alpha \lambda_{L} K_{\beta} K_{\gamma} K_{\delta}\left[-\beta\left(1+l^{2} K_{\gamma}\right) \frac{t_{4}}{t_{3}^{ \pm 1}}+\gamma\left(1+l^{2} K_{\beta}\right) \frac{t_{4}}{t_{2}^{ \pm 1}}\right] \\
& +\beta K_{\beta}\left[\left(\gamma^{2}-\xi^{2}\right)+l^{2} K_{\gamma}^{2}\right] \mu \delta P\left(\frac{t_{1}}{t_{3}}\right)^{ \pm 1}-\gamma K_{\gamma}\left[\left(\beta^{2}-\xi^{2}\right)+l^{2} K_{\beta}^{2}\right] \mu \delta P\left(\frac{t_{1}}{t_{2}}\right)^{ \pm 1} \\
\quad= & 4 \alpha \beta \gamma \delta \mu \xi^{2}\left(K_{\beta}-K_{\gamma}\right)
\end{aligned}
$$

where $K_{\beta}=\beta^{2}+\xi^{2}, K_{\gamma}=\gamma^{2}+\xi^{2}, K_{\delta}=\delta^{2}+\xi^{2}, P=-\left(C_{1}^{2} / C_{2}^{2}\right)\left(\xi^{2}+\alpha^{2}\right)+2 \xi^{2}, t_{1}=S_{1} / C_{11}$, $t_{2}=S_{2} / C_{22}, t_{3}=S_{3} / C_{3}$ and $t_{4}=S_{4} / C_{4}$.

Here "+" sign corresponds to skew symmetric and "-" sign refers to symmetric modes of the Lamb waves. 
In the absence of the liquid layer, $\left(\lambda_{L} \rightarrow 0\right)$, Eq. (2.10) reduces to

$$
\beta K_{\beta}\left[\left(\gamma^{2}-\xi^{2}\right)+l^{2} K_{\gamma}^{2}\right]\left(\frac{t_{1}}{t_{3}}\right)^{ \pm 1}-\gamma K_{\gamma}\left[\left(\beta^{2}-\xi^{2}\right)+l^{2} K_{\beta}^{2}\right]\left(\frac{t_{1}}{t_{2}}\right)^{ \pm 1}=\frac{4 \alpha \beta \gamma \xi^{2}\left(K_{\beta}-K_{\gamma}\right)}{P}
$$

This equation is the same as the obtained and studied by the authors (Sharma and Kumar, 2014) for Lamb waves in an isotropic elastic plate within the couple stress theory.

\section{Numerical results and discussion}

To investigate the effects of characteristic length and inner microstructure on the propagation of Lamb waves, the material of the plate is assumed to have properties similar to cortical bone, so following Vavva et al. (2009), the material properties are Young's modulus $E=\mu(3 \lambda+2 \mu) /(\lambda+\mu)=14 \mathrm{GPa}$, Poisson ratio $\nu=\lambda /[2(\lambda+\mu)]=0.37$ and density $\rho=1500 \mathrm{~kg} / \mathrm{m}^{3}$, the values of bulk longitudinal and shear velocities are $C_{1}=4063 \mathrm{~m} / \mathrm{s}$, $C_{2}=1846 \mathrm{~m} / \mathrm{s}$, respectively. The fluid medium used is an inviscid liquid with $C_{L}=1.5 \cdot 10^{3} \mathrm{~m} / \mathrm{s}$ and density $\rho_{L}=1000 \mathrm{~kg} / \mathrm{m}^{3}$. The size of bone internal microstructure (internal cell size) ranges from 10 to $500 \mu \mathrm{m}$. Here, to study the impact of characteristic length, different cases of characteristic lengths $l$, comparable with the internal cell size $h$ such as $l=0.0003 \mathrm{~m}, l=0.0001 \mathrm{~m}$, $l=0.00003 \mathrm{~m}$ are considered.

Figures 2 and 3 show the phase velocity profile of Lamb waves in an elastic plate under the effect of an inviscid liquid layer on both sides for a fixed characteristic length $(l=0.0001 \mathrm{~m})$ and fixed thickness of the liquid layer $(H=0.02 \mathrm{~m})$. Figure 2 shows the phase velocity profiles of fundamental modes of symmetric (S0) and skew symmetric (A0) Lamb waves. The phase velocity profiles of skew symmetric Lamb waves for different modes $(M=1, M=2$ and $M=3)$ are shown in Fig. 3. It is observed that in the wave number range 0-2, the secular equation has only one root - these are the fundamental modes ( $\mathrm{S} 0$ and $\mathrm{A} 0$ ) of Lamb waves. As the wave number increases, new roots start appearing for both symmetric and skew symmetric modes. The behaviour of these profiles is quite in agreement with the earlier findings. It is observed that the magnitude of phase velocity of these profiles is quite high for the lower wave number, which decreases at a steady rate and becomes asymptotic for higher wave numbers.

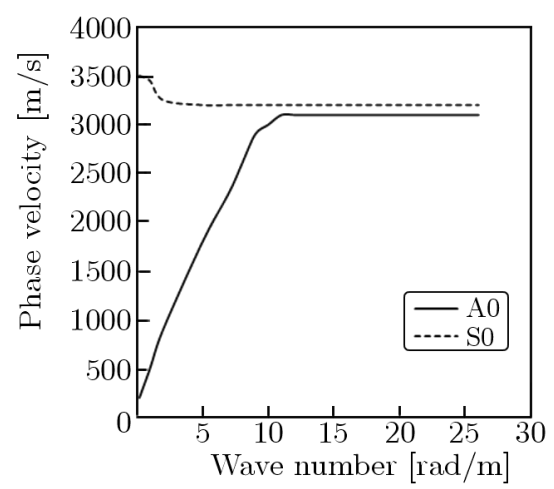

Fig. 2. Phase velocity profile of fundamental modes of Lamb waves (symmetrical (S0) and skew symmetrical $(\mathrm{A} 0)$ ) with the wave number in an elastic plate bordered with a liquid layer

Lamb waves propagating in a plate bounded by a liquid on both sides leak some of its energy into the liquid and this situation has practical importance in the field of NDT. To study the impact of loadings on Lamb waves, Figs. $4 \mathrm{a}$ and $4 \mathrm{~b}$ are drawn showing phase velocity profiles of skew symmetrical Lamb waves for two different values of thickness of the liquid layer $(\mathrm{H} 1=0.01 \mathrm{~m}$ and $\mathrm{H} 2=0.02 \mathrm{~m})$, and the profiles are compared with the case of no liquid 


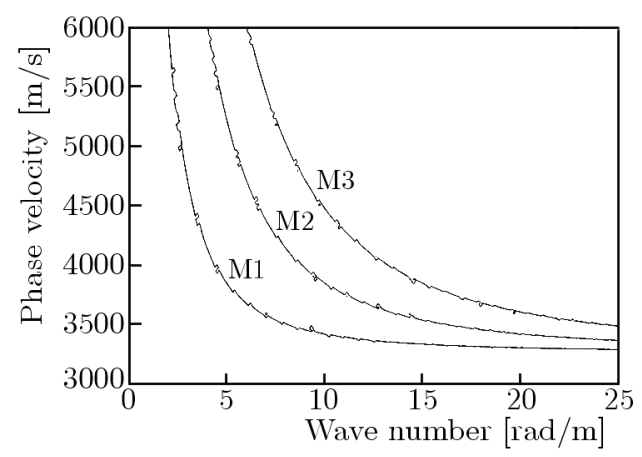

Fig. 3. Phase velocity profiles of skew symmetrical modes (M1, M2 and M3 for $\mathrm{M}=1,2,3$, respectively) of Lamb waves with the wave number in an elastic plate bordered with a liquid layer

loadings H0. Figure 4a is for $\mathrm{M}=1$ skew symmetrical mode and Fig. $4 \mathrm{~b}$ is for $\mathrm{M}=2$ mode. In both cases, the characteristic length $(l=0.0001 \mathrm{~m})$ is kept fixed. It can be seen that with an increase in the thickness of the liquid loadings, the phase velocity of the skew symmetrical Lamb waves decreases for the same wave number. As the variation in the phase velocity is very small with the increasing value of thickness of the liquid layer and it is not possible to show these variations graphically for a wide range of wave numbers, so the figures are drawn with very small variations in the wave numbers to depict the effects more precisely.

(a)

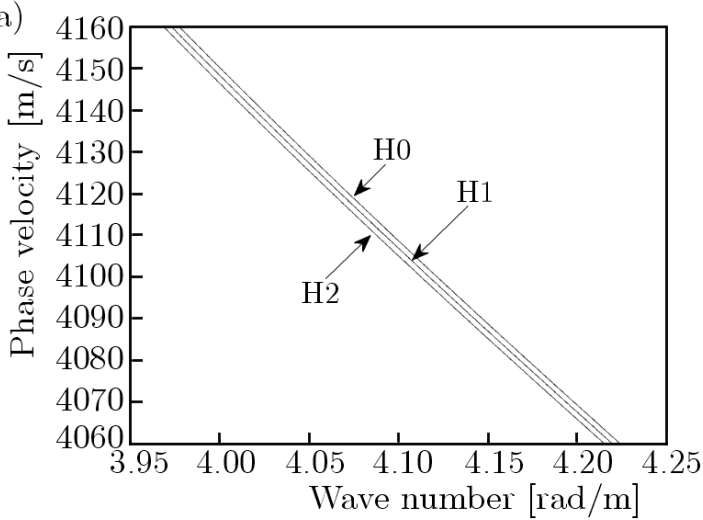

(b)

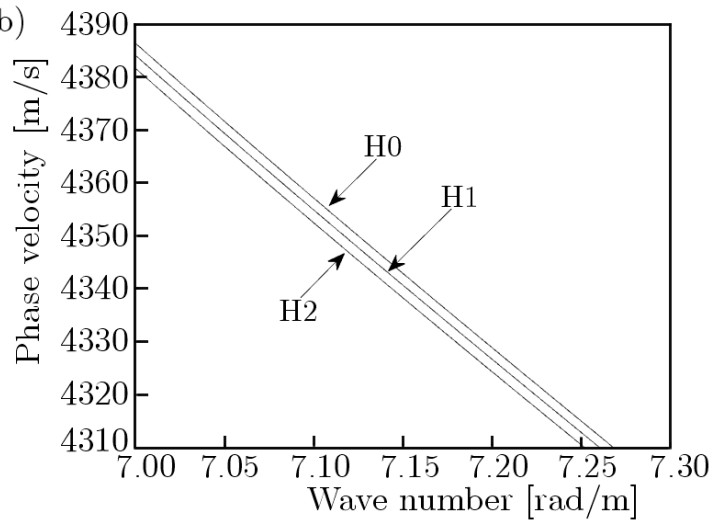

Fig. 4. Phase velocity profile of Lamb waves for skew symmetrical mode ((a) $M=1$, (b) $M=2$ ) with the wave number in an elastic plate bordered with a liquid layer of different thicknesses $(\mathrm{H} 0=0.00 \mathrm{~m}$, $\mathrm{H} 1=0.01 \mathrm{~m}$ and $\mathrm{H} 2=0.02 \mathrm{~m}$ )

The effect of the characteristic length parameter $l$, on the phase velocity profiles of skew symmetric Lamb waves are shown in Figs. 5a and 5b. The characteristic length parameter $l$ is a microstructural parameter involved in the couple stress theory and is assumed to be of the order of internal cell size of the considered material. The material of the considered plate has properties similar to cortical bone and the internal cell size of cortical bone varies from 10 to $500 \mu \mathrm{m}$ (Vavva et al., 2009). So, here three different values of characteristic lengths are considered, for profile L1, the characteristic length is $0.00003 \mathrm{~m}$, for L2 profile, the characteristic length is $0.0001 \mathrm{~m}$ and for L3, it is $0.0003 \mathrm{~m}$. In all the three cases, the considered characteristic length of the material lies in the range of internal cell size of the material. As the problem deals with the study of microstructural effects and to observe these effects again, graphs are drawn again with a very small variation in the wave number and phase velocity of Lamb waves. Figure 5a shows the phase velocity profiles of skew symmetric Lamb waves for $\mathrm{M}=1$ mode, with three different values of the characteristic length and fixed thickness of the liquid layer $(\mathrm{H}=0.02 \mathrm{~m})$. Figure $5 \mathrm{~b}$ shows the phase velocity profiles of skew symmetric Lamb waves for $\mathrm{M}=2$ mode, again with 

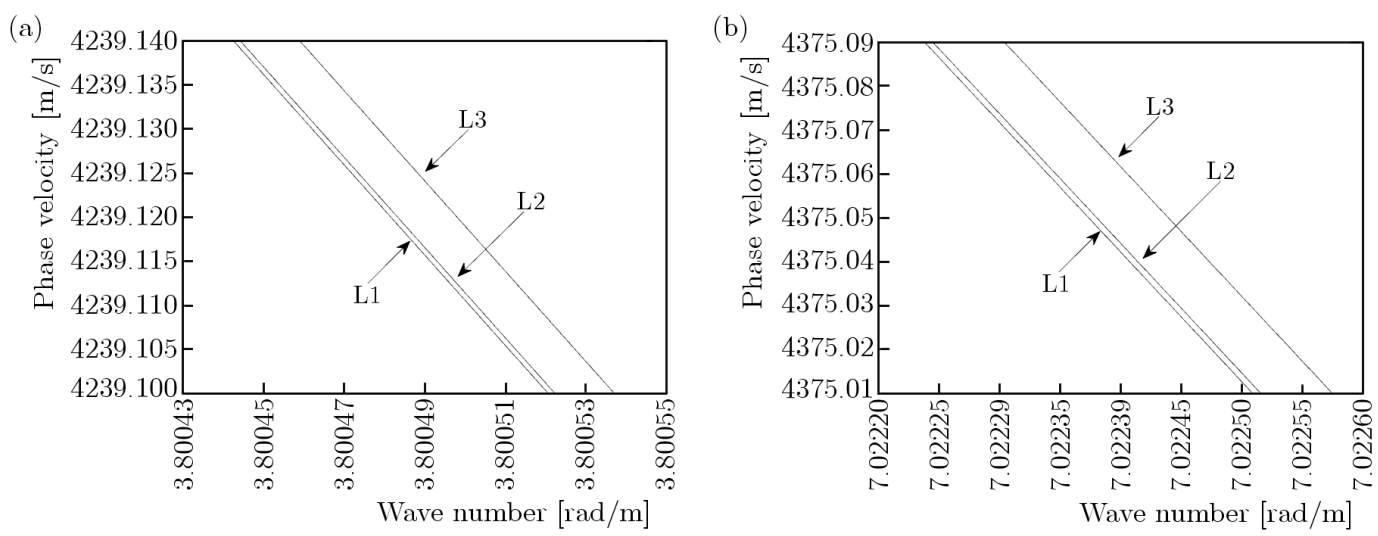

Fig. 5. Phase velocity profile of Lamb waves for skew symmetrical mode ((a) $M=1$, (b) $M=2$ ) with the wave number in an elastic plate bordered with a liquid layer of fixed thickness and different characteristic lengths $\mathrm{L} 1=0.00003 \mathrm{~m}, \mathrm{~L} 2=0.0001 \mathrm{~m}$ and $\mathrm{L} 3=0.0003 \mathrm{~m}$

the same considered values of the characteristic length and fixed thickness of the liquid layer $(\mathrm{H}=0.02 \mathrm{~m})$. From both figures, it is observed that with an increase in the characteristic length parameter $l$, the phase velocity of skew symmetric Lamb waves is also increasing for the same wave number.

\section{Conclusion}

As the physical model of the problem consists of a thin plate loaded with an inviscid liquid on both sides, it is of practical use in ultrasonic immersion testing of plates. The impact of liquid loadings is studied on the propagation of phase velocity of Lamb waves. Three different cases for thickness of the liquid loaded on both sides of the plate are considered. It is observed that with an increase in the thickness of the loadings, the phase velocity tends to decrease. The material of the plate considered is assumed to have properties similar to cortical bone (Vavva et al., 2009), so it also enhances the applicability of this model in characterisation of the properties of bones loaded with different type of fluids.

To find the impact of the microstructural parameter, the problem is solved by applying the couple stress theory (Hadjesfandiari and Dargush, 2011). This theory involves only one microstructural parameter, called the characteristic length $(l)$ and is assumed to be of the order of internal cell size of the material (Georgiadis and Velgaki, 2003; Vavva et al., 2009). Here, three different values of the characteristic length are considered. They are comparable with the internal cell size of the material and their effect is studied on the phase velocity of the Lamb waves. It is observed that with an increase in the value of this parameter, the phase velocity of the Lamb wave also increases.

\section{References}

1. Akgoz B., Civalek O., 2013, Modeling and analysis of micro-sized plates resting on elastic medium using the modified couple stress theory, Meccanica, 48, 863-873

2. Chen W., Li X., 2014, A new modified couple stress theory for anisotropic elasticity and microscale laminated Kirchhoff plate model, Archive of Applied Mechanics, 84, 323-341

3. Cosserat E., Cosserat F., 1909, Théorie des corps déformables (Theory of Deformable Bodies), A. Hermann et Fils, Paris 
4. Das T.K., Sengupta P.R., Debnath L., 1991, Thermo-visco-elastic Rayleigh waves under the influence of couple stress and gravity, International Journal of Mathematics and Mathematical Sciences, 14, 3, 553-560

5. ERIngen A.C., 1968, Theory of micropolar elasticity, [In:] Fracture, Liebowitz H. (Ed.), vol. 2, Academic Press, New York, 662-729

6. Georgiadis H.G., Velgaki E.G., 2003, High-frequency Rayleigh waves in materials with microstructure and couple-stress effects, International Journal of Solids and Structures, 40, 2501-2520

7. Hadjesfandiari A.R., Dargush G.F., 2011, Couple stress theory for solids, International Journal of Solids and Structures, 48, 2496-2510

8. Koiter W.T., 1964, Couple stresses in the theory of elasticity, I and II, Proceedings of the Koninklijke Nederlandse Akademie Van Wetenschappen B, 67, 17-44

9. LAKES R.S., 1991, Experimental micro mechanics methods for conventional and negative Poisson's ratio cellular solids as Cosserat continua, Journal of Engineering Materials and Technology, 113, $148-155$

10. Lakes R.S., Yoon H.S., Katz J.L., 1986, Ultrasonic wave propagation and attenuation in wet bone, Journal Biomedical Engineering, 8, 143-148

11. Lamb H., 1917, On waves in an elastic plate, Proceedings of the Royal Society of London, 114-128

12. Mindlin R.D., 1964, Micro-structure in linear elasticity, Archive for Rational Mechanics and Analysis, 16, 51-78

13. Mindlin R.D., Tiersten H.F., 1962, Effects of couple-stresses in linear elasticity, Archive for Rational Mechanics and Analysis, 11, 415-488

14. NowACKI W., 1974, Micropolar Elasticity, International Center for Mechanical Sciences, Courses and Lectures No. 151, Udine, Springer-Verlag, Wien-New York

15. Osborne M.F.M., Hart S.D., 1945, Transmission, reflection and guiding of an exponential pulse by a steel plate in water, I: theory, Journal of the Acoustical Society of America, 17, 1-18

16. Ottosen N.S., RistinmaA M., Luung C., 2000, Rayleigh waves by the indeterminate couplestress theory, European Journal of Mechanics - A/Solids, 19, 929-947

17. SсHоch V.A., 1952, Der schalldurchgang durch platten (Sound transmission in plates), Acoustica, 2, $1-17$

18. Sengupta P.R., Ghosh B., 1974, Effects of couple stresses on the propagation of waves in an elastic layer, Pure and Applied Geophysic, 112, 331-338

19. Sharma J.N., Kumar S., 2009, Lamb waves in micropolar thermoelastic solid plates immersed in liquid with varying temperature, Meccanica, 44, 305-319

20. Sharma V., Kumar S., 2014, Velocity dispersion in an elastic plate with microstructure: effects of characteristic length in a couple stress model, Meccanica, 49, 1083-1090

21. Sharma J.N., Pathania V., 2003, Generalized thermoelastic Lamb waves in a plate bordered with layers of inviscid liquid, Journal of Sound and Vibration, 268, 897-916

22. Toupin R.A., 1962, Elastic materials with couple-stresses, Archive for Rational Mechanics and Analysis, 11, 385-414

23. Vavva M.G., Protopappas V.C., Gergidis L.N., Charalambopoulos A., Fotiadis D.I., Polyzos D., 2009, Velocity dispersion of guided waves propagating in a free gradient elastic plates: application to cortical bone, Journal of the Acoustical Society of America, 125, 5, 3414-3427

24. Viktorov I.A., 1967, Rayleigh and Lamb Waves, Plenum Press, New York 
25. Voigt W., 1887, Theoretische Studien fiber die Elastizitatsverhiltnisse der Kristalle (Theoretical studies on the elasticity relationships of crystals), Abh Gesch Wissenschaften, $\mathbf{3 4}$

26. Wu J., Zhu Z., 1992, The propagation of Lamb waves in a plate bordered with layers of a liquid, Journal of the Acoustical Society of America, 91, 861-867

27. Wu Z., Ma X., Brett P.N., Xu J., 2009, Vibration analysis of submerged rectangular microplates with distributed mass loading, Proceedings of the Royal Society A, 465, 2104, 1323-1336

Manuscript received October 2, 2014; accepted for print May 10, 2015 